\section{SHAHEEN PALLIATIVE CARE PROJECT, PAKISTAN}

Sobia Bilal, Maimoona Ali, Sadaf Mudassir, Awais Naeem, Adeel Basharat Hussain, Jalil Khan, Qamar Abbas, Mohammad Ayyaz Butt. Shaheen Palliative Care Project

\subsection{6/spcare-2021-PCC.102}

Background Shaheen Palliative care project team aim to develop a training and service oriented programme. The team help provide compassionate palliative care for patients living with a life-limiting illness and their families, through physical, emotional, spiritual, psychological and social support. There are no current palliative care services in hospitals or communities for the people of Pakistan. The aim of the team is to equip and train Pakistani healthcare workers with the essential knowledge and skills to able to provide the best quality palliative care aswell as public awareness of palliative medicine.

Methods A service evaluation about palliative care services in Pakistan. Two surveys were sent, one to all grades of doctors at two hospital sites in Peshawar, Pakistan and the second to members of the general public. The data was then analysed using Excel.

Results There were 60 respondents, in total, to both the surveys. $95 \%$ were involved in care of the terminally ill with only $20 \%$ of them being confident in managing patients with a life limiting disease. $85 \%$ said they did not have access to necessary drugs for pain control in terminally ill patients. $70 \%$ thought hospice was synonymous with palliative care. $100 \%$ of respondents thought there is a need for palliative care services in Pakistan.

Conclusions This evaluation was undertaken to help develop a training and service-oriented palliative care programme for people of Pakistan working towards specialist palliative care services. The Shaheen Palliative care team have set up the first Palliative Care Postgraduate certificate at Khyber University, October 2019 and the National University of Medical Sciences, Rawalpindi, shall also be starting Post Graduate Palliative Care Certificate from end of 2020. The multi-professional international team are leading the way in developing new services and palliative medicine education in Pakistan for both the healthcare professional and the public.

\section{Posters $85-86$ | pain}

\section{MORE THAN A GUT FEELING: A LITERATURE REVIEW OF MANAGING PAIN ASSOCIATED WITH OESOPHAGEAL STENTING}

Louisa Nelms, Jon Tomas. Queen Elizabeth Hospital Birmingham

\subsection{6/spcare-2021-PCC.103}

Background Oesophageal cancer has a 5-year survival rate of $17 \%$. Adequate symptom management is hence important at all stages of disease. Dysphagia, commonly experienced by these patients, can have a detrimental impact on quality of life. This can be managed by endoscopic insertion of an oesophageal stent aimed at improving symptoms. This procedure, however, is frequently associated with side effects, most notably chest pain.

Methods A literature review of the natural history of chest pain following insertion of oesophageal stent and management options was undertaken. Databases, including Medline and Embase, were searched using 'o/esophageal stent' and 'stent/ chest pain' as well as associated terms. Some 160 articles were found. Non-relevant, non-English, and duplicate papers were discarded. Sixteen articles were retained and examined in detail, particularly regarding the incidence and management of stent-associated pain.

Results Chest pain following stent insertion is common, with reported incidence ranging from 9-60\%. Often worse within the first 48 hours, this pain typically improves over time. Pain still requiring analgesia, however, has been reported over four weeks post-procedure. The most common treatment described is opioids. One study found that $40 \%$ of patients required an opiate to manage pain (mean daily dose of $43 \mathrm{mg}$ ). Overall the studies lacked detail about effective dosing regimens and long term follow up. The evidence for other treatment modalities was weak and often based on single case studies. These included radiotherapy, sublingual glyceryl trinitrate and, phrenic nerve block. Stent removal may be warranted, but is associated with risks of endoscopy and failure.

Conclusions Chest pain following oesophageal stenting is common and may persist despite treatment. Pain has a major impact on these patients' quality of life; effective management regimes are essential. Further studies are needed to evaluate long term consequences for patients and better explore appropriate therapeutic options for this symptom.

\section{CASE REPORT: RESPIRATORY DEPRESSION FOLLOWING A SWITCH IN OXYCODONE PREPARATIONS TREATED WITH SUBCUTANEOUS NALOXONE}

Mairéad Doherty, Maeve O'Reilly, Marie Twomey. St Luke's Hospital, Rathgar

\subsection{6/spcare-2021-PCC.104}

Background Oxycodone/naloxone has a reported equivalent analgesic effect to oxycodone due to insufficient naloxone reaching systemic circulation to affect analgesia. Naloxone plasma concentrations can increase in some, e.g. patients with hepatic or renal impairment.

Case presentation A 75 year-old male with prostate and lung carcinoma was admitted for palliative radiotherapy. Admission analgesia included oxycodone/naloxone $70 / 35 \mathrm{mg}$ bd, pregabalin $100 \mathrm{mg}$ bd and oxynorm $20 \mathrm{mg}$ prn.

Management Day 3: Multiple breakthroughs required for severe pain. Oxycodone/naloxone changed to oxycodone 90 $\mathrm{mg}$ bd (maximum licensed dose of oxycodone/naloxone exceeded). Pregabalin increased to $150 \mathrm{mg}$ bd.

Day 4: Respiratory infection diagnosed and treated with intravenous antibiotics. Mild myoclonus but no other signs of toxicity. Pain ongoing.

Day 5: Sudden deterioration with respiratory depression (respiratory rate - 6, oxygen saturations -60\%). Rapid response to naloxone 40 micrograms intravenously. Opioids and pregabalin discontinued. Multiple further naloxone doses required for recurrent respiratory depression. Continuous subcutaneous infusion (CSCI) of $1.5 \mathrm{mg}$ naloxone commenced.

Day 6: Naloxone infusion discontinued when pain recurred. CSCI of oxycodone $30 \mathrm{mg} / 24$ hours commenced and subsequently rotated to hydromorphone $4 \mathrm{mg} / 24$ hours due to mild opioid toxicity. 